\title{
An optimal charging algorithm to minimise solid electrolyte interface layer in lithium-ion battery
}

\author{
M S S Malik ${ }^{\mathrm{a}}$, Guang $\mathrm{Li}^{\mathrm{a}, *}$, Zheng Chen ${ }^{\mathrm{a}}$ \\ ${ }^{a}$ School of Engineering and Materials Science, Queen Mary University of London, E1 4NS, United Kingdom
}

\begin{abstract}
This article presents a novel control algorithm for online optimal charging of lithium-ion battery by explicitly incorporating degradation mechanism into control, to reduce the degradation process. The health of battery directly relates to degradation and capacity fade in cycles of charging. We mainly focus on the growth of the solid electrolyte interface (SEI) layer, which is the primary source of degradation of batteries. This article addresses the challenge of minimising SEI layer growth during charging by incorporating the first-order SEI layer growth rate model into a non-linear model predictive control approach. A single particle model (SPM) is used for optimal charging using orthogonal projection-based model reformulation. Gauss pseudo-spectral method is used for the optimisation of charging trajectories. Results of the optimal algorithm are compared with the traditional constant current constant voltage $(\mathrm{CCCV})$ approach without considering SEI layer growth. It is ensured that overpotential caused by lithium plating remains in a healthy regime which is another feature of the proposed strategy. Simulation results are presented to demonstrate the advantages of the proposed charging method.
\end{abstract}

Keywords: Optimal Charging, Non-linear model predictive control, lithium-ion battery, Pseudo-spectral methods

\section{Introduction}

This paper proposes a non-linear model predictive control (NMPC) framework to extend the life of a lithium-ion battery 3 by decreasing the growth rate of the solid electrolyte interface (SEI) layer during charging. Due to the advantages including 4 high energy density, low self-discharge rate and low maintenance requirements, lithium-ion batteries have been used as energy 5 storage components in many applications, such as electric vehicles (EVs). Long charging time of EVs is one of the hurdles 6 for EV applications. However, fast charging at higher rates can accelerate the degradation process of batteries, reduce their power and capacity limits. Conventional charging methods include constant current (CC), constant voltage (CV), multi-stage constant current, pulse, variable current and constant current constant voltage (CCCV) charging strategies [1]. All of these charging strategies are simple to implement, but they cannot explicitly deal with with the ageing of batteries. In order to reduce the effect of capacity fade, optimal charging strategies are proposed to deal with the state of health (SOH) of batteries [3. Off-line and online optimal charging strategies are proposed in which the influence of battery SOC, charging current and charging profile are closely related to capacity fade.

The motivation of this article stems from the need to explicitly incorporate the degradation effects in battery charging in a manner that minimises charging time while ensuring safety, reliability and reducing the degradation process. Excessive

${ }^{*}$ Corresponding author

Email addresses: msajjad.sabir@uettaxila.edu.pk (M S S Malik), g.li@qmul.ac.uk (Guang Li ), chen@kust.edu.cn (Zheng Chen ) 
battery damage and ageing of battery systems can be reduced by imposing predefined limits on various states or variables such as current rates, state of charge (SOC) and temperature. The popular adopted CCCV charging method cannot guarantee the satisfaction of these limits if the charging current is too high. In contrast, it is possible to charge batteries more aggressively using model-based control algorithms based on electrochemical models while not causing damage and increasing degradation. Such algorithms rely on electrochemical models instead of equivalent circuit models because the equivalent circuit models cannot cope with the constraints on the internal variables. The existing model-based optimal charging algorithms include dynamic programming [4, genetic algorithms [6, 7] and pseudo-spectral optimisation [5, 8]. One drawback of the model-based controller is the heavy computational burden because of the complexities of the electrochemical models. Researchers tried to reduce the computational burden by introducing efficient algorithms, e.g. 9. The primary advantage of the model-based controller is to directly constrain unwanted reactions, associated with degradation. Secondly, a model-based controller can adapt to parametric variations in the battery, and thereby quantifies the ageing of the battery and explicitly measures SOH 2]. These variations represent the dynamics of lithium-ion batteries with specific accuracy and computational efficiency. In this paper, we incorporate the SEI layer growth model to an optimal charging control algorithm so that an optimal trade-off between charging time and growth of SEI layer film subject to constraints can be achieved.

This work can be seen as an extension of published work on health-conscious NMPC of lithium-ion batteries [9], where the effect of lithium plating into online optimal charging of battery management system (BMS). Battery charge/trajectory was expressed in terms of one flat output trajectory to reduce computational burden by a factor of 5 compared with pseudospectral optimisation alone. Moreover, the proposed constant current constant side reaction overpotential (CCC $\eta$ ) strategy ensured the side reaction constraint to remain in healthy regime during charging. However, in [9], authors considered only one side reaction with no quantification of $\mathrm{SOH}$. Optimal charging trajectories are calculated in a healthy regime without estimation of degradation effects [10, 11]. Moreover, researchers also proposed a health-aware fast-charging methodology using model predictive control. For example, [12] explored moving horizon approach incorporating chemical degradation effects, but the SEI film resistance is not estimated. Due to ageing effects, battery SOH does not follow a specified trajectory. This deviation is minimised by proposing the balancing control method [13]. The effect of temperature and degradation effects also alter the voltage and state of energy responses [14. All of the above research works do not contribute to the quantification of the ageing effect represented by the thickness of the SEI layer.

The main contribution of this paper compared to the previously published works is that the proposed method explicitly minimises the effect of SEI layer growth in charging, thus reducing the capacity fade of battery. SEI layer is passively formed on negative electrode due to side reaction, and the main factor causing capacity fade for a battery 10, 15. Lithium intercalation in a battery, during charging, increases the volume of graphite particles [22]. This volume change stretches the surface film on the edges, which has limited flexibility, resulting in the surface film to break. It changes the order of film passivity and exposes more carbon to the electrolyte. This will act as a barrier for lithium-ion intercalation, which introduces capacity fade effects. Therefore, incorporating SEI layer dynamics will help us to minimise the capacity degradation effect. The proposed online methodology controls the growth of the SEI layer during charging using a single-particle model (SPM) and Gauss pseudo-spectral method. SPM is an appropriate candidate because it gives a satisfactory trade-off between accuracy and computational efficiency compared to other electrochemical models in the literature. Dynamics of battery electrode 

is

in SPM is approximated by a linear combination of Legendre polynomials and unknown time coefficients. The number of Legendre polynomials depends on the best fit of the electrode trajectory compared to experimental results. Even polynomials automatically satisfy the electrode's condition at the centre of the particle, thus reducing the complexity of the electrochemical model [17]. The non-convex behaviour of the resulting model-based optimal control is computationally challenging, even for low order models. Dynamic programming and other optimisation algorithms can solve non-convex problems, but they have the drawback of high computational cost, which makes them unsuitable for online control applications. A pseudo-spectral method is used to optimise the charging trajectory because it has high convergence rates, which makes it computationally efficient. Also, it can solve non-linear and non-convex optimisation problems effectively [25]. The pseudo-spectral method transforms a continuous problem into a non-linear programming (NLP) problem, which can be efficiently solved using efficient commercial software.

This paper's novel and unique contribution is the development of a NMPC framework which accounts for chemical degradation along-with side reaction overpotential. In the proposed strategy, a dynamic model predicts the future responses of a controlled plant. These future predictions are computed by minimising performance cost, defined in terms of states and input sequences. The concept of the receding horizon is introduced to realise online optimisation. The proposed strategy ensures that physicsbased constraint must be satisfied during the whole process of charging. The NMPC framework's optimisation results are compared with the traditional CCCV approach to show the advantages of the proposed strategy.

The remainder of this article is organised as follows: Section 2 presents the governing equations and reformulated SPM. Theory of SEI layer is presented in Section 3 while Gauss pseudo-spectral method is briefly discussed in Section 4. Section 5 presents the problem formulation while Section 6 explains the mathematical formulation of NMPC strategy to find an optimal solution. Section 7 shows the results of battery optimal control problem and compares it to the conventional CCCV charging method. Section 8 concludes the article.

\section{Single particle model}

In this section, the governing equations of SPM are presented. Orthogonal projection techniques are used to convert partial differential equations to ordinary differential equations[16]. A physics-based SPM is used in this work to achieve a trade-off between accuracy and computational efficiency [17, 18. The parameters of SPM and reference potential curves for both electrodes are obtained from [19]. Assumptions for modelling SPM can be found in [10].

\subsection{SPM equations}

Fick's second law of diffusion gives information about solid-phase diffusion dynamics. The governing differential equation

$$
\begin{gathered}
\frac{\partial c_{s}(r, t)}{\partial r}=\frac{D_{s}}{r^{2}} \frac{\partial}{\partial r}\left(r^{2} \frac{\partial c_{s}(r, t)}{\partial r}\right) \\
\left.\frac{\partial c(r, t)}{\partial r}\right|_{r=0}=0 \\
\left.\frac{\partial c(r, t)}{\partial r}\right|_{r=R}= \pm \frac{J(t)}{F D_{s} a}
\end{gathered}
$$


where $c_{s}, D_{s}, a$ and $J$ are the solid-state concentration, diffusion constant, interfacial surface area and molar flux of lithium-ion of corresponding electrode (negative or positive) respectively. $F$ is Faraday's constant and $R$ is the radius of the corresponding electrode. Interfacial area of electrode can be defined as

$$
a=\frac{3 \epsilon}{R}
$$

where $\epsilon$ is porosity of electrode. The molar flux of lithium-ions $J_{i}$ are defined as

$$
\begin{aligned}
& J_{n}(t)=-\frac{I(t)}{S L_{n}} \quad \text { (Negative Electrode) } \\
& J_{p}(t)=\frac{I(t)}{S L_{p}} \quad(\text { Positive Electrode })
\end{aligned}
$$

where $S$ and $L_{j}$ are sheet area and length of electrode respectively. $I(t)$ is applied current (positive for charging). The bulk state of charge (SOC) is defined as

$$
S O C(t)=\frac{c_{s, a v g}(t)}{c_{s, \max }}
$$

where $c_{s, a v g}(t)$ is the average lithium-ions concentration of the electrode, i.e.

$$
c_{s, a v g}(t)=\int_{0}^{R} c_{s} d r
$$

and $c_{s, \max }$ is the maximum concentration of lithium ions of the electrode. The surface SOC is defined as

$$
\operatorname{SOC}^{\operatorname{surf}}(t)=\frac{c_{s, a v g}^{\text {surf }}(t)}{c_{s, \text { max }}}
$$

The relationship between the molar flux of lithium-ion and the potential difference between solid and solution phases in any electrode, can be expressed as

$$
\begin{aligned}
& J(t)=i_{0}(t)\left[\exp \left(\frac{\alpha_{a} F}{R_{g} T} \eta(t)\right)-\exp \left(\frac{\alpha_{c} F}{R_{g} T} \eta(t)\right)\right] \\
& i_{0}(t)=a k\left(c_{s, \text { max }}-c_{s}^{\text {surf }}\right)^{\alpha_{a}}\left(c_{s}^{\text {surf }(t)}\right)^{\alpha_{c}} c_{e}^{\alpha_{a}}
\end{aligned}
$$

$k$ is the reaction rate constant, and $i_{0}$ is the current density of the respective electrode. $\alpha_{a}$ and $\alpha_{c}$ are electrode transfer coefficients in anode and cathode, respectively. $c_{s, \max }$ and $c_{s}^{\text {surf }}$ are maximum and surface concentrations in the corresponding electrode respectively. $c_{s}^{\text {surf }}$ can be computed as $c(R, t) . R_{g}$ is gas constant, and $T$ is the temperature which is $298 \mathrm{~K}$ in this work. The overpotential $\eta$ is defined as the difference between solid and electrolyte potential and it can be expressed for a negative electrode as

$$
\eta_{n}(t)=\phi_{1, n}(t)-\phi_{2, n}(t)-U_{n}\left(S O C_{n}^{\text {surf }}(t)\right)
$$


where $\phi_{1, n}$ is solid-phase potential while $\phi_{2}$ is solution-phase potential. The potential drop in the solution phase between two electrodes is

$$
\phi_{2, p}(t)-\phi_{2, n}(t)=I(t) R_{c e l l}
$$

where the subscripts $p$ and $n$ refer to positive and negative electrodes, and $R_{\text {cell }}$ is the resistance of cell which is a lumped parameter. The potential difference between positive and negative electrodes is defined as cell voltage. It can be expressed using (10), 12) and (13) as

$$
V_{\text {cell }}(t)=\eta_{p}-\eta_{n}+U_{p}-U_{n}+I(t) R_{\text {cell }}
$$

$U$ is an open circuit potential of the corresponding electrode. During lithium plating, side reaction affects the negative electrode while charging [2]. The overpotential due to side reaction can be written as follows

$$
\eta_{s r}=\phi_{1, n}-\phi_{2, n}
$$

To avoid excessive lithium plating, batteries should operate satisfying $\eta_{s r} \geq 0$, i.e. the desired regime.

\subsection{Model Reformulation}

Diffusion equation (1) needs to be reformulated into an ordinary differential equation. Galerkin method with Legendre spatial basis functions is used to discretise Fick's law. The reformulated SPM reduces the dynamics using three state variables. The entire process is briefly described below, and further details can be found in [20.

It is assumed that concentration in any electrode is only a function of time and radial periphery. The lithium-ion concentration $c(r, t)$ can be approximated by a linear combination of Legendre polynomials and corresponding time variables:

$$
c(r, t) \approx \sum_{i=0}^{M} \phi_{i}(r) \beta_{i}(t) \approx \phi_{0}(r) \beta_{0}(t)+\phi_{2}(r) \beta_{2}(t)+\phi_{4}(r) \beta_{4}(t)+\phi_{6}(r) \beta_{6}(t)
$$

where $\phi_{i}(r)$ is Legendre polynomial, and $\beta_{i}(t)$ is the unknown time coefficient of any electrode. The boundary condition at centre of particle (2) are automatically satisfied because of even Legendre polynomials , $i=0,2,4,6$. The Legendre polynomials can be normalised:

$$
\int_{0}^{R} \phi_{i}(r) \phi_{j}(r) d r=\left\{\begin{array}{ccc}
0 & \text { if } & i \neq j \\
1 & \text { if } & i=j
\end{array}\right.
$$

where $R$ is the radius of a particle. Substituting equation (16) into (1) gives

$$
\sum_{i=0}^{4} \phi_{i}(r) \dot{\beta}_{i}=D_{s}\left[\frac{2}{r} \sum_{i=0}^{4} \frac{d \phi_{i}(r)}{d r} \beta_{i}(t)+\sum_{i=0}^{4} \frac{d^{2} \phi_{i}(r)}{d r^{2}} \beta_{i}((t)]\right.
$$


where $\dot{\beta}_{i}$ is differentiation of $\beta_{i}$ with respect to time. The diffusion dynamics can then be expressed as follows

$$
\left[\begin{array}{c}
\beta_{\dot{0}}(t) \\
\beta_{\dot{2}}(t) \\
\beta_{\dot{4}}(t) \\
\beta_{\dot{6}}(t)
\end{array}\right]=\frac{D_{s, n}}{R_{n}^{2}}\left[\begin{array}{cccc}
0 & 9 \sqrt{5} & 20 & 29.4 \sqrt{13} \\
0 & 0 & 35 \sqrt{5} & 16.8 \sqrt{65} \\
0 & 0 & 0 & 46.2 \sqrt{13} \\
0 & 0 & 0 & 0
\end{array}\right]\left[\begin{array}{c}
\beta_{0}(t) \\
\beta_{2}(t) \\
\beta_{4}(t) \\
\beta_{6}(t)
\end{array}\right]
$$

Similarly the boundary condition at $r=R$ can be expressed as

$$
\frac{3}{R} \sqrt{\frac{5}{R}} \beta_{2}+\frac{10}{R} \sqrt{\frac{9}{R}} \beta_{4}+\frac{21}{R} \sqrt{\frac{13}{R}} \beta_{6}= \pm \frac{J(t)}{D_{s} a F}
$$

where \pm is used according to negative or positive electrode formulation. After applying orthogonal projection techniques, considering the second boundary condition; dynamics of coefficients $\left[\beta_{0}, \beta_{2}, \beta_{4}, \beta_{6}\right]^{T}$ can be obtained. $\beta_{6}$ does not have dynamics, and thus can be discarded. The final dynamics is derived, using $(18)$ and $(19)$, in the form of a state-space model.

$$
\begin{aligned}
& \dot{x}=A x+B u \\
& y=C x+D u
\end{aligned}
$$

where the state vector is defined as $x=\left[\beta_{0}, \beta_{2}, \beta_{4}\right]^{T}$ and the input $u$ is current $I(t)$. Outputs of the system can be computed algebraically.

\section{Modelling of SEI layer}

Research on side reactions in lithium-ion batteries are mainly focused on passive film formation on the negative electrode. During charging, increment in volume recorded due to the increase in space between the graphene planes [21]. This means the SEI film has significant porosity which leads to the conclusion that it grows as a result of solvent diffusion 22, 23. The first principle of the SEI film growth model is taken from [15], which is caused by the effect of slow solvent diffusion/reduction near the surface of the negative electrode. Assumptions on parameter values and modelling fundamentals can be seen in [15, 24]. In contrast to the SPM model described in section II, certain changes in the negative electrode are proposed. Firstly equivalent molar flux at a negative electrode is equal to intercalation $\left(J_{n}\right)$ plus side reaction $\left(J_{s}\right)$.

$$
J_{e q, n}=J_{n}+J_{s}
$$

Moreover the equation of overpotential 12 is expressed by

$$
\eta_{n}=\phi_{1, n}-\phi_{2, n}-U_{n}-\frac{J_{e q, n}}{a_{n}} R_{f i l m}
$$


where $R_{f i l m}$ is the resistance of the SEI film. Side reaction molar flux can be expressed as

$$
J_{s}=-i_{o, s} a_{n} e^{-\frac{R_{g} T \eta_{s}}{2 F}}
$$

where $i_{o, s}$ is the exchange current density for side reaction, and $\eta_{s}$ is the side reaction overpotential, represented as

$$
\eta_{s}=\phi_{1, n}-\phi_{2, n}-U_{r e f, s}-\frac{J_{e q, n}}{a_{n}} R_{f i l m}
$$

where $U_{r e f, s}$ is open circuit potential for side reaction and is equal to $0.4 \mathrm{~V}$ [15. For the first cycle, film resistance, $R_{f i l m}$, is defined as

$$
R_{f i l m}=R_{S E I}+R_{p}(t)
$$

where $R_{S E I}$ is initial film resistance, $0.01 \Omega m^{2}$ in this work, while $R_{p}(t)$ is the resistance of the products formed during charging and is defined as

$$
R_{p}(t)=\frac{\delta_{\text {film }}}{\kappa_{p}}
$$

where $\kappa_{p}$ is the conductivity of the electrolyte. The mathematical expression of the rate of SEI film resistance $\left(\delta_{f i l m}\right)$ is written as

$$
\frac{\partial \delta_{f i l m}}{\partial t}=-\frac{J_{s} M_{p}}{a_{n} \rho_{p} F}
$$

where $M_{p}, \rho_{p}$ are molecular weight and density of products formed during side reaction, respectively. From (25)-(27), time rate change of SEI film resistance is written as

$$
\frac{\partial R_{f i l m}}{\partial t}=\frac{i_{o, s} M_{p}}{\kappa_{p} \rho_{p} F} e^{-\frac{R_{g} T \eta_{s}}{2 F}}
$$

The above equation represents the dynamics of SEI film resistance.

\section{Gauss Pseudo-spectral Method}

Implementation of online optimal charging strategies based on electrochemical battery models can be challenging due to two reasons: 1) online method can be computationally expensive, 2) problem is non-linear, particularly due to constraints. This section describes briefly about Gauss pseudo-spectral method (GPM) which is employed as an efficient optimization method to resolve the online optimization problem. The first requirement in GPM is to change the time interval from arbitrary bounds $t \in\left[t_{0}, t_{f}\right]$ to the interval $\tau \in[-1,1]$ by

$$
t=\frac{\left(t_{f}-t_{0}\right) \tau+\left(t_{f}+t_{0}\right)}{2}
$$

The Legendre-Gauss (LG) collocation points used in GPM are all interior to the interval $[-1,1]$ [25]. The initial point $\tau_{0}=0$ and final point $\tau_{f}=1$ are also taken into account. The Lagrange interpolating polynomials at a set of collocation points are 
building blocks for the integration approximation matrix in (33). These polynomials can be expressed as [26].

$$
L_{k}(t)=\frac{w(t)}{\left(t-t_{k}\right) w^{\prime}\left(t_{k}\right)}
$$

where $t_{1}, t_{2}, \ldots$ are the roots of polynomial while $w(t)$ is a Gauss weight and defined as

$$
w(t)=\prod_{i=1}^{N}\left(t-t_{i}\right)
$$

A function can be approximated using Lagrange interpolation formula as

$$
f(t) \approx \sum_{k=1}^{N} f\left(t_{k}\right) L_{k}(t)
$$

The dynamic constraints are discretised using an integration approximation matrix:

$$
X\left(t_{i}\right)=X\left(t_{0}\right)+\frac{t_{f}-t_{0}}{2} \sum_{k=1}^{N} A_{i k} f\left(X\left(t_{k}\right), t_{k}\right), \quad i=1, \ldots ., N
$$

where $t_{i}(s)$ are set of collocation points, $A_{i k}$ is the integral approximation matrix and $X\left(t_{0}\right)=x_{0}$ is the initial value. The approximation of function $(32)$ is simplified due to a unique property of Lagrange polynomials, expressed as

$$
L_{j}\left(\tau_{i}\right)=\left\{\begin{array}{ccc}
0 & \text { if } & i \neq j \\
1 & \text { if } & i=j
\end{array}\right.
$$

The elements in the integral approximation matrix for Gauss points can be approximated by using Axelsson's algorithm [27.

$$
A_{i k}=\frac{w_{i}}{2}\left(1+t_{i}+\sum_{v=1}^{n-2} P_{v}\left(t_{k}\right)\left[P_{v+1}\left(t_{i}\right)-P_{v-1}\left(t_{i}\right)\right]+P_{N-1}\left(t_{k}\right)\left[P_{N}\left(t_{i}\right)-P_{N-2\left(t_{i}\right)}\right]\right)
$$

where $w_{i}$ is the $i_{t h}$ Gauss weight and $P_{j}$ is the $j_{t h}$ Legendre polynomial. Finally, cost $J$ can be approximated using pseudospectral transcription [28:

$$
J=\Phi\left(X\left(t_{f}\right), t_{f}\right)+\frac{t_{f}-t_{0}}{2} \sum_{k=1}^{N} g\left(X_{k}, U_{k}, t_{k}\right) w_{k}
$$

where $\Phi$ relates to terminal condition and $g($.$) is a function of state, input and time at k_{t h}$ collocation point. The non-linear boundary constraints can be approximated as

$$
\phi\left(X\left(t_{0}\right), t_{0}, X\left(t_{f}\right), t_{f}\right)=0
$$

The final state $X\left(t_{f}\right)$ is defined as

$$
X\left(t_{f}\right)=X\left(t_{0}\right)+\frac{t_{f}-t_{0}}{2} \sum_{k=1}^{N} w_{k} f\left(X_{k}, U_{k}, \tau_{k}\right),
$$


The above equation is a Gauss quadrature approximation to the continuous definition of final states. The cost function can be discretised using the quadrature rule, written as

$$
\int_{a}^{b} f(t) d t \approx \sum_{i=1}^{N} \alpha_{i} f\left(t_{i}\right)
$$

where $\alpha_{i}$ and $t_{i}$ are $i_{t h}$ quadrature weight and point (or node) respectively. The cost (35) and non-linear boundary constraints (36) form the NLP which can be solved by mature optimization routines.

\section{Problem Formulation}

The main aim of this work is to minimise SEI film resistance during charging. We adopt the receding horizon control concept to conduct online optimization at each sampling time. The optimization problem to be resolved is expressed as

$$
\begin{aligned}
& \underset{I(t)}{\operatorname{minimize}} \int_{t_{o}}^{t_{f}}\left[\left(S O C_{n}(t)-S O C_{r e f}\right)^{2}+q R_{f i l m}^{\prime}(t)\right] d t \\
& \text { subject to } \\
& \text { model } E q \cdot(7)-(12),(16), \\
& 0 \leq I(t) \leq I_{\text {max }}, \\
& 0 \leq V(t) \leq V_{\text {max }}, \\
& \eta_{s r} \geq 0
\end{aligned}
$$

where $q$ is control parameter, $R_{f i l m}^{\prime}(t)$ is the time rate change of film resistance, $S O C_{r e f}$ is reference state of charge, $\eta_{s r}$ is side reaction overpotential, and $I_{\max }, V_{\max }$ are the maximum current and voltage respectively. The goal of this problem is to charge the cell to the desired SOC, $S O C_{r e f}$, and minimize the growth of SEI film resistance. Also, the battery should operate in the healthy regime defined by the constraints on current, voltage and overpotential. Please note that side reaction over-potentials $\eta_{s}$ and $\eta_{s r}$ are not similar. $\eta_{s}$ is side reaction based upon diffusion of the organic solvent present in electrolyte, while $\eta_{s r}$ is due to the lithium plating which should be greater than or equal to zero for the safe operation of the battery. State of charge in a negative electrode can be written, using equation (7), as

$$
S O C_{n}(t)=\frac{\phi_{0}(r) \beta_{0}(t)+\phi_{2}(r) \beta_{2}(t)+\phi_{4}(r) \beta_{4}(t)+\phi_{6}(r) \beta_{6}(t)}{c_{\max , n}}
$$

$R_{f i l m}^{\prime}(t)$ is a function of intercalation and side reaction over-potentials in the negative electrode 28 . Equating 22 and (24), we get

$$
\eta_{s}=\eta_{n}+U_{n, r e f}-U_{r e f, s}
$$


where $\eta_{n}(t)$ can be written, using (5), (10) and $(12)$, as

$$
\eta_{n}(t)=\frac{R_{\text {gas }} T}{F} \ln \left(\frac{J_{n}(t)}{i_{o, n}(t) a_{n}}\right)
$$

Overpotential is a function of surface concentration $c_{n}^{\text {surf }}(t)$, which can be approximated by

$$
c_{n}^{\text {surf }}(t)=\phi_{0}(R) \beta_{0}(t)+\phi_{2}(R) \beta_{2}(t)+\phi_{4}(R) \beta_{4}(t)+\phi_{6}(R) \beta_{6}(t)
$$

Finally $R_{f i l m}^{\prime}(t)$ can be formulated as

$$
\begin{aligned}
& R_{\text {film }}^{\prime}(t)=X \exp \{-\left(\frac{R_{\text {gas }} T}{2 F}\right)\left[\left(\frac{R_{\text {gas }} T}{F}\right)\right. \\
& \ln \left(\frac{-I(t)}{A_{n} L_{n} k_{n} \sqrt{\phi_{0}(R) \beta_{0}(t)+\phi_{2}(R) \beta_{2}(t)+\phi_{4}(R) \beta_{4}(t)+\phi_{6}(R) \beta_{6}(t)-c_{\text {max }, n}}}\right. \\
&\left.\frac{1}{\sqrt{\phi_{0}(R) \beta_{0}(t)+\phi_{2}(R) \beta_{2}(t)+\phi_{4}(R) \beta_{4}(t)+\phi_{6}(R) \beta_{6}(t)} \sqrt{c_{e}}}\right)+ \\
&\left.\left.U_{n, r e f}\left(\frac{\phi_{0}(R) \beta_{0}(t)+\phi_{2}(R) \beta_{2}(t)+\phi_{4}(R) \beta_{4}(t)+\phi_{6}(R) \beta_{6}(t)}{c_{\text {max }, n}}\right)-0.4\right]\right\}
\end{aligned}
$$

where $X$ is a constant with value of $5.4 \times 10^{-10}$ [15]. Moreover, pseudo-spectral method discretise the cost function (39) using (35) to form a NLP problem. The proposed approach is compared against the optimal charging method without incorporating the SEI layer growth rate [9, 10, which aims to resolve the online optimization problem:

$$
\begin{aligned}
& \underset{I(t)}{\operatorname{minimize}} \int_{t_{o}}^{t_{f}}\left(S O C_{n}(t)-S O C_{r e f}\right)^{2} d t \\
& \text { subject to } \\
& \text { model } E q \cdot 77-12,16, \\
& 0 \leq I(t) \leq I_{\text {max }}, \\
& 0 \leq V(t) \leq V_{\text {max }}
\end{aligned}
$$

\section{Non-linear model predictive control strategy}

In this section, we address how to formulate and implement the NMPC control algorithm based on the optimization problem set up in Section 5

\subsection{Prediction}

A dynamic model predicts future responses of the controlled plant. The system can be represented as a discrete state-space representation form as

$$
\mathbf{x}(k+1)=A \mathbf{x}(k)+B \mathbf{u}(k)
$$

where $\mathbf{x}(k)$ and $\mathbf{u}(k)$ are prediction model state and input vectors at $k_{t h}$ sampling instant respectively. $A$ and $B$ are system matrices. The prediction of states is generated by solving the model over $N$ sampling intervals (prediction horizon), generating 
an optimal control sequence. Define the state and input sequences for $N$ steps as

$$
\mathbf{u}(k)=\left[\begin{array}{c}
u(k \mid k) \\
u(k+1 \mid k) \\
u(k+2 \mid k) \\
\cdot \\
\cdot \\
u(k+N-1 \mid k)
\end{array}\right], \quad \mathbf{x}(k)=\left[\begin{array}{c}
x(k+1 \mid k) \\
x(k+2 \mid k) \\
x(k+3 \mid k) \\
\cdot \\
\cdot \\
x(k+N \mid k)
\end{array}\right]
$$

where $u(k+j \mid k)$ and $x(k+j \mid k)$ denote input and state at time $k+j$, predicted at time $k$ respectively. It means that $x(k+j \mid k)$ evolves according to the prediction model as

$$
x(k+j+1 \mid k)=A x(k+j \mid k)+B u(k+j \mid k), \quad j=0,1,2, \ldots \ldots
$$

with initial condition defined as $x(k \mid k)=x(k)$. In this particular work, there are three states and one input, as discussed in subsection 2.2. The final dynamic prediction model in the form of state space is shown below

$$
\left[\begin{array}{c}
\dot{\beta}_{0}(t) \\
\dot{\beta}_{2}(t) \\
\dot{\beta_{4}}(t)
\end{array}\right]=\left[\begin{array}{ccc}
0 & 10.15 & -20.80 \\
0 & -11.35 & 23.26 \\
0 & -13.96 & -62.42
\end{array}\right]\left[\begin{array}{c}
\beta_{0}(t) \\
\beta_{2}(t) \\
\beta_{4}(t)
\end{array}\right]+10^{-3} \times\left[\begin{array}{c}
0.22 \\
0.28 \\
0.35
\end{array}\right] I(t)
$$

whereas time coefficient $\beta_{6}(t)$ is redundant because of zero dynamics, but must be known to find the state of charge of battery. It can be algebraically calculated using the following output equation.

$$
\left[\begin{array}{l}
\beta_{0}(t) \\
\beta_{2}(t) \\
\beta_{4}(t) \\
\beta_{6}(t)
\end{array}\right]=\left[\begin{array}{ccc}
1 & 0 & 0 \\
0 & 0 & 1 \\
0 & -0.088 & -0.39
\end{array}\right]\left[\begin{array}{c}
\beta_{0}(t) \\
\beta_{2}(t) \\
\beta_{4}(t)
\end{array}\right]+\left[\begin{array}{c}
0 \\
0 \\
0 \\
0.22
\end{array}\right] I(t)
$$

Finally, from section 4 we know that, the output of the system is approximated by

$$
\beta_{j}(\tau) \approx \boldsymbol{\beta}_{\boldsymbol{j}}(\tau)=\sum_{k=0}^{N} L_{k}(\tau) \beta_{j}\left(\tau_{k}\right)
$$

where $\beta_{j}$ is the corresponding output in any electrode and $L_{k}(\tau)$ is the Lagrange polynomial.

\subsection{Optimisation}

The future predictions are computed by minimising predicted performance cost, defined in terms of states and inputs sequences. Cost function $J(k)$ as defined in (35), is a function of $\mathbf{u}(k)$ and optimal input sequence for the problem denoted as $u^{*}(k)$. It can be written as

$$
\mathbf{u}^{*}(k)=\arg \min _{\mathbf{u}} J(k)
$$


In this work, the cost function as defined in (39), is discretised using Legendre Gauss quadrature rule ( 38$)$. It can be written

$$
\begin{array}{r}
J=\int_{t_{o}}^{t_{f}}\left[\left(S O C_{n}(t)-S O C_{r e f}\right)^{2}+q R_{f i l m}^{\prime}(t)\right] d t \\
J \approx \frac{t_{f}-t_{0}}{2} \sum_{i=1}^{N}\left[w_{i}\left(S O C_{n}\left(\tau_{i}\right)-S O C_{r e f}\right)^{2}+q R_{f i l m}^{\prime}\left(\tau_{i}\right)\right]
\end{array}
$$

where $w_{i}$ is Gauss weight and computed using 31 . $R_{f i l m}^{\prime}\left(\tau_{i}\right)$ can be solved using 45 . State of charge at Legendre Gauss point is written as

$$
S O C_{n}\left(\tau_{i}\right)=\frac{\phi_{0}(r) \beta_{0}\left(\tau_{i}\right)+\phi_{2}(r) \beta_{2}\left(\tau_{i}\right)+\phi_{4}(r) \beta_{4}\left(\tau_{i}\right)+\phi_{6}(r) \beta_{6}\left(\tau_{i}\right)}{c_{\max , n}}
$$

\subsection{Receding horizon implementation}

In all of the future optimal input sequence $\mathbf{u}^{*}(k)$, only the first value is taken as a input to the plant:

$$
u(k)=u^{*}(k \mid k)
$$

The process of evaluating $\mathbf{u}^{*}(k)$ and implementing the first element of $\mathbf{u}^{*}$ is then repeated at each sampling instance $k=$ $0,1,2, \ldots$. Due to this repetition of prediction at every instance, it is known as an online optimisation. The prediction horizon keeps its constant length throughout the optimisation process, and therefore the approach is known as a receding horizon strategy. In this work, the term $\left(t_{f}-t_{0}\right)$ denotes the prediction horizon as seen in the above discretised cost function (54). Future $\mathrm{N}$ states $\left[\beta_{0}(t), \beta_{2}(t), \beta_{4}(t)\right]^{T}$ and $\mathrm{N}$ inputs $I(t)$ are predicted at a current sampling instant. In the next sampling instant, $t_{f}-t_{0}$ will be the same as in last instant but initial values of the system is changed.

\subsection{Constraints}

Apart from any obvious equality constraints that satisfy the dynamics of the model, every control problem encounters inequality constraints on input and state variables. As noted from the problem (39), one input and two non-linear constraints are part of this optimisation exercise. The linear inequality constraint is of the form

$$
A_{e q} \mathbf{x} \leq b_{e q}
$$


Using eq. (33), we get the following form of linear inequality constraint

$$
\left[\begin{array}{cccc}
I_{N} & -T t \times A(1,2) \times M_{N} & -T t \times A(1,3) \times M_{N} & -T t \times B(1,1) \times M_{N} \\
\mathbf{0}_{N} & I_{N}-T t \times A(2,2) \times M_{N} & -T t \times A(2,3) \times M_{N} & -T t \times B(2,1) \times M_{N} \\
\mathbf{0}_{N} & -T t \times A(3,2) \times M_{N} & I_{N}-T t \times A(3,3) \times M_{N} & -T t \times B(3,1) \times M_{N}
\end{array}\right]\left[\begin{array}{c}
\beta_{0_{N \times 1}} \\
\beta_{2_{N \times 1}} \\
\beta_{4_{N \times 1}}
\end{array}\right]<=
$$

where $I_{N}, \mathbf{0}_{N}$ are $N \times N$ identity and zero matrices respectively. $M_{N}$ is an integration approximation matrix of order $N \times N$, as defined in (34). $A$ and $B$ are system matrices, taken from 20 . According to Gauss pseudo-spectral notation, $x_{j_{N \times 1}}$ means $\left[x_{j}\left(\tau_{1}\right), x_{j}\left(\tau_{2}\right), x_{j}\left(\tau_{3}\right), ., ., ., x_{j}\left(\tau_{N}\right)\right]^{T}$. This size of $A_{e q}$ and $b_{e q}$ matrices depends on the chosen prediction horizon. The two non-linear constraints are voltage $V_{\text {cell }}$ and side reaction overpotential $\eta_{s r}$; both are function of states and input. In pseudo-spectral notation, it can be written as

$$
\left.V\left(\tau_{i}\right)\right)=\frac{R_{\text {gas }} T}{F}\left[\ln \left(\frac{J_{p}\left(\tau_{i}\right)}{i_{o, p}\left(\tau_{i}\right) a_{p}}\right)-\ln \left(\frac{J_{n}\left(\tau_{i}\right)}{i_{o, n}\left(\tau_{i}\right) a_{n}}\right)\right]+U_{p}\left(\operatorname{SOC}_{p}^{r e f}\left(\tau_{i}\right)\right)-U_{n}\left(\operatorname{SOC}_{n}^{\text {ref }}\left(\tau_{i}\right)\right)+I\left(\tau_{i}\right) R_{\text {cell }}
$$

$$
\eta_{s r}\left(\tau_{i}\right)=\frac{R_{g a s} T}{F} \ln \left(\frac{J_{n}\left(\tau_{i}\right)}{i_{o, n}\left(\tau_{i}\right) a_{n}}\right)+U_{n}\left(\operatorname{SOC}_{n}^{\text {ref }}\left(\tau_{i}\right)\right)
$$

Finally, the algebraic cost (54), along with linear (57) and non-linear constraints $(59,60)$ make up the NLP problem. It is further solved by MATLAB function "fmincon" in this work.

\section{Results and Discussion}

The initial state of charge (SOC) is set to 0.4 while two upper current limits are considered in this work, i.e. $I_{\max }=5 \mathrm{~A}$ and $7 A$. Reference state of charge, $S O C_{r e f}$ is taken as 0.96 , and the voltage limit is set to 4.2 volts. Problem (39) is set to start at $t_{o}=0$, using a NMPC approach. At each time step, the solver predicts future instances with a prediction horizon of $100 s$ using four collocation points. The initial guess of the solution at the present time step is a solution at the last sampling instance.

State of charge in the negative electrode is shown in Figure 1(a) and (d) for $I_{\max }=5 A$ and $7 A$, respectively. SOC is compared for two charging methods: the proposed method (39) and the method (46) presented in [10]. It can be seen that the reference SOC is achieved in each methodology but charging time in the proposed optimal case is higher than the method (46) for both upper limits of current. In the case of $I_{\max }=5 \mathrm{~A}$, a $9.6 \%$ increase in charging time is recorded in the proposed method (39) while at $I_{\max }=7 \mathrm{~A}$, charging time difference is $22 \%$. The higher difference in case of $I_{\max }=7 \mathrm{~A}$ is understandable 
because the CCCV methodology charges the battery in constant current (CC) scenario for maximum time. As we increase the maximum current for both methodologies, the difference in charging time becomes larger. Note that longer charging time using the proposed SEI optimal charging method can significantly reduce the SEI layer growth as demonstrated below.

It is a known practice that CCCV charging terminates at fairly low current i.e. $5 \mathrm{~mA}$ or $50 \mathrm{~mA}$. In this work, the later current value is used. In Figure 1(a), SOC reaches the reference value at $t_{1}$ while it stays at same value until $t_{2}$. This is due to the current profile in CCCV charging. The optimal CCCV algorithm charges the battery using constant current (CC) approach from $t=0$ to $t=t_{1}$. At $t_{1}$, it switches to constant voltage $(\mathrm{CV})$ approach which means current needs to be lower down to keep the voltage constant. Reference SOC is achieved at $t_{1}$ which needs to be same till $t_{2}$. Current value drops from $I_{\max }$ to $50 m A$ in time span of $t_{1}-t_{2}$.

Figure 1(b) and (e) depict the results of optimal CCCV charging for $I_{\max }=5 \mathrm{~A}$ and $7 \mathrm{~A}$, respectively. CCCV charging splits

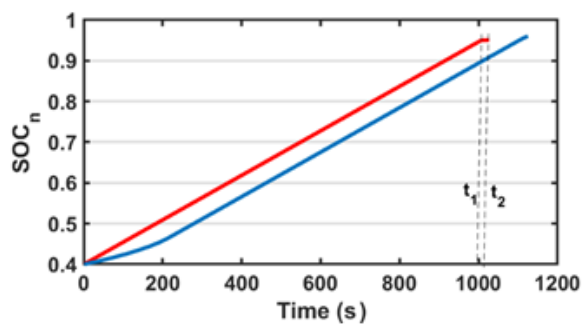

(a)

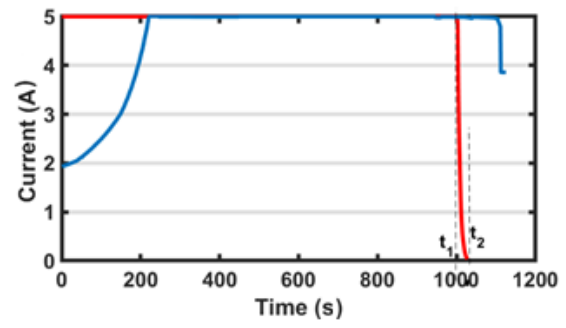

(b)

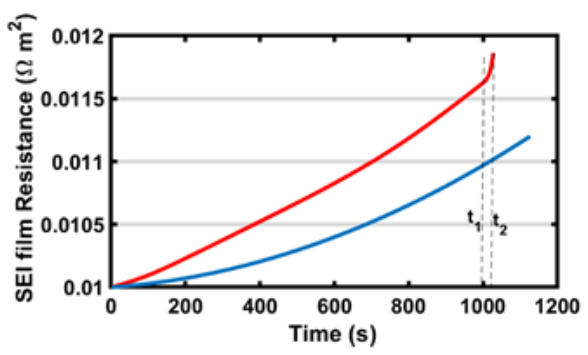

(c)

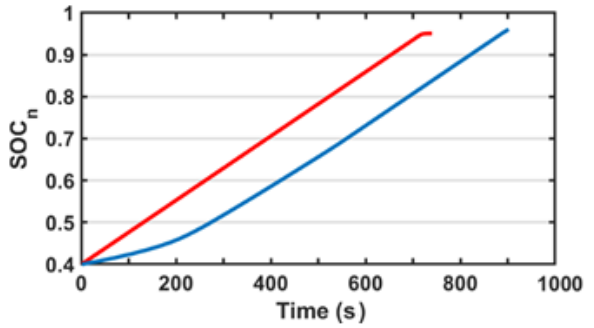

(d)

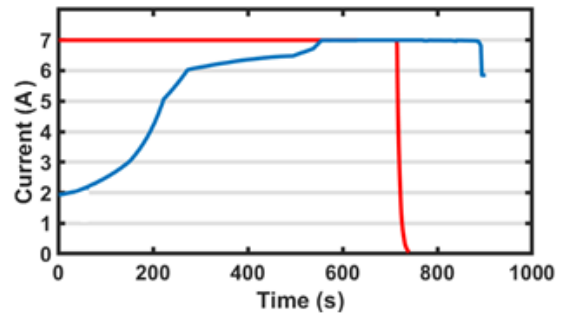

(e)

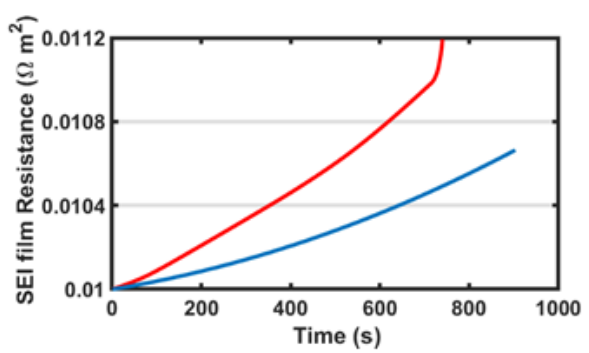

(f)

Figure 1: Comparison of State of charge $(\mathrm{a}, \mathrm{d})$, current profile $(\mathrm{b}, \mathrm{e})$ and SEI film resistance $(\mathrm{c}, \mathrm{f})$ vs charging time at current upper bounds of $I_{\max }=5 A(\mathrm{a}-\mathrm{c})$ and $I_{\max }=7 A(\mathrm{~d}-\mathrm{f}) ;$ Optimal CCCV charging $(-)$, Proposed SEI optimal charging $(-)$

into two phases; CC from $t=0$ to $t=t_{1}$ and CV from $t=t_{1}$ to $t=t_{2}$. In both cases for SEI reduction optimal charging method, charging starts at fairly low value to reduce the rate of lithium plating. This is because, at low SOCs, the reference potential of the negative electrode is quite high, i.e. the possibility of $\eta_{s r}$ to be negative. Thus to be in the healthy regime, the charging current needs to increase slowly, since higher current can lead to lower resistance of the SEI layer. The proposed SEI reduction optimal charging profile finishes, in either of the maximum current limits, at higher current value as compared to the optimal CCCV charging method. Termination of SEI optimal charging at higher current value has two advantages, 
(i) it compensates for charging time which considerably increases due to low SOC regime (where $\eta_{s r}$ can be negative), and (ii) It reduces the growth of SEI layer which increases at low values of current.

The growth of SEI film resistance is shown in Figure 1(c) and (f) for $I_{\max }=5 \mathrm{~A}$ and $7 \mathrm{~A}$, respectively. It is evident from the figures that SEI film resistance is quite high in optimal CCCV and SEI optimal charging cases when maximum current is $5 A$. The primary reason is that lower current takes more time to complete charging. SEI film resistance drops significantly in proposed SEI optimal charging compared to the optimal CCCV charging at given maximum current value. The overall optimal charging time increases but SEI film resistance decreased. This can be explained as at higher SOCs the algorithm uses a maximum value of current, so that molar flux is high. In case of $I_{\max }=5 \mathrm{~A}$ (Figure 1(e)), SEI layer resistance is recorded as $0.0118 \Omega \mathrm{m}^{2}$ in optimal CCCV case, reduced to $0.0112 \Omega \mathrm{m}^{2}$ in proposed SEI optimal formulation scenario. The percentage increase of SEI layer resistance from initial value $\left(0.01 \Omega m^{2}\right)$ is $18 \%$ and $12 \%$ in optimal CCCV and the proposed SEI reduction optimal charging method, respectively, which represents a $5.2 \%$ decrease of SEI layer growth using the proposed charging method. Lower percentage difference is recorded $(4.95 \%)$ in case of $I_{\max }=7 \mathrm{~A}($ Figure $1(\mathrm{f}))$ between two strategies. The main difference is in the final phase of optimal CCCV charging, where it uses low current as compared to the proposed optimal approach. The maximum value of surface concentration and negative $\eta_{s r}$ increase the SEI layer resistance to a fairly high value in optimal CCCV approach.

The profile of SEI layer in optimal CCCV charging can be categorised on the basis of SOC regimes, i.e. low or high. At low SOC regime, the current is high, which acts as a source of lithium plating. At maximum current, overpotential of negative electrode $(43)$ is high, which makes side reaction overpotential $\eta_{s r}(60)$ negative. This is not desirable as only positive $\eta_{s r}$ guarantees the reduction of lithium plating side effects. Termination of optimal CCCV charging usually happens at low current, which is $50 \mathrm{~mA}$ in this work. Due to low current at the end stage of charging $\left(t_{1}\right.$ to $\left.t_{2}\right)$, side reaction overpotential for SEI layer $\eta_{s}(24)$ is very low. The exponential term in (28) ultimately leads to spike in SEI layer profile at the final stage of optimal CCCV charging.

Another added advantage of the proposed algorithm is to charge batteries in the healthy regime, which means side reaction overpotential of lithium plating is positive during the whole process. Note that cost function does not always guarantee the desired result. It can be argued that side reaction overpotential will always be positive to decrease the value of the exponential term in the cost function. However, this cannot be extrapolated for an entire range of possible values of current which indicates that limit on $\eta_{s r}$ is necessary in this work. Figure 2 shows side reaction overpotential $\eta_{s r}$ in optimal CCCV and SEI optimal charging. It is evident from the figure that in optimal CCCV charging, $\eta_{s r}$ is negative while it is positive in proposed SEI optimal charging, successfully avoiding lithium plating. Hence, It means that the proposed charging algorithm runs in a healthy regime along with reducing SEI film resistance.

Total charging time and maximum charging current value affect the resistance of the SEI layer growth. In optimal CCCV charging, higher current upper bound means fast charging and lesser growth of the SEI layer. However, the optimal algorithm makes sure that SEI layer resistance is as low as possible along-with successfully avoiding lithium plating during the whole process of charging. If the current upper bound is constant, charging time is higher in the SEI optimal charging case than optimal CCCV but quite low SEI film resistance. Next, we compare the two methods in two scenarios to investigate: $(i)$ At what conditions, is charging time for both methodologies the same? (ii) If SEI film resistance is the same, how does charging 


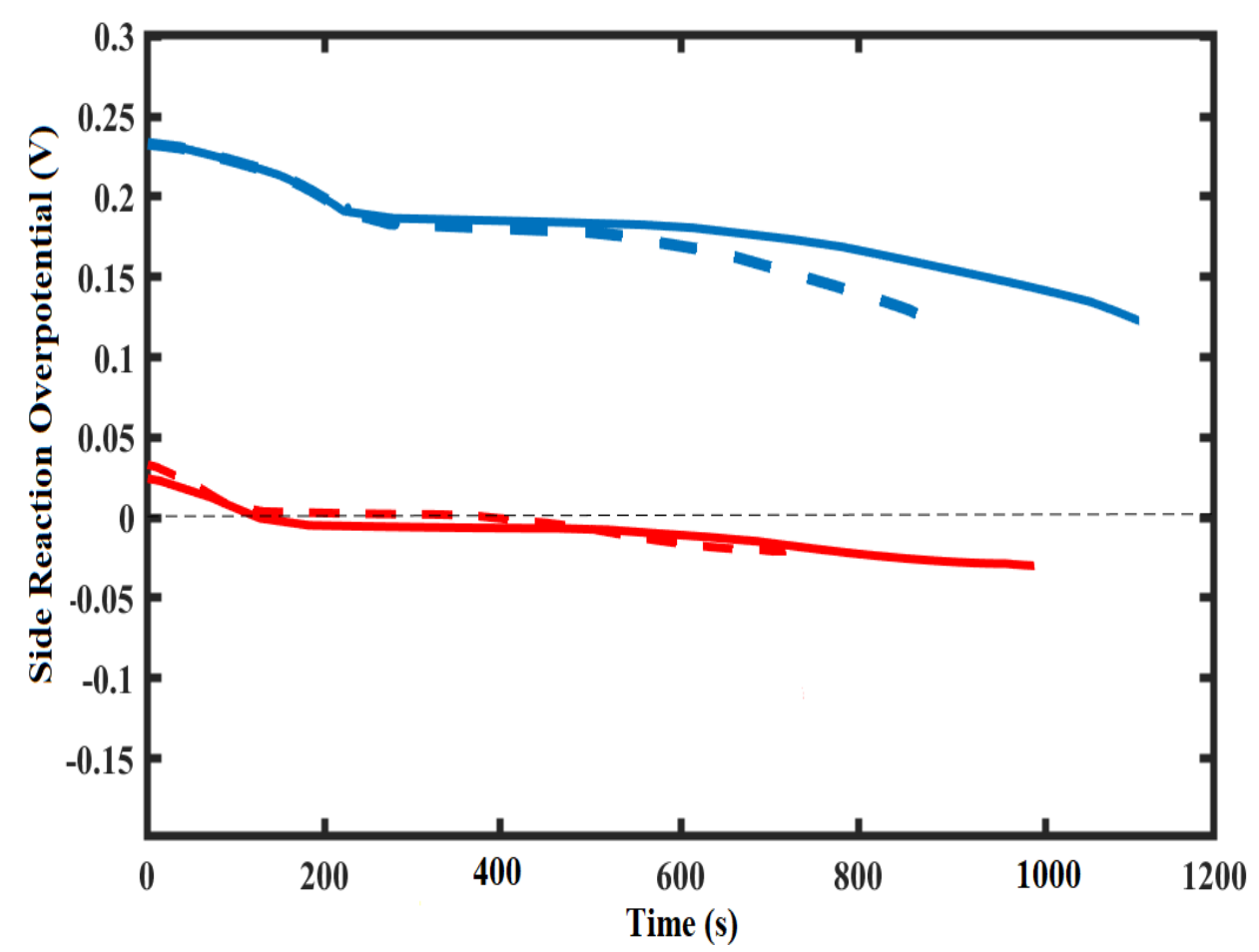

Figure 2: Relationship between charging time and side reaction overpotential $\left(\eta_{s r}\right)$ in the proposed SEI optimal charging $(-)$ and optimal CCCV charging $(-)$ approaches, $I_{\max }=5 A($ solid $)$ and $I_{\max }=7 \mathrm{~A}$ (dashed)

time relate to current upper bound?

\subsection{Scenario I: Same Charging time}

In optimal CCCV strategy, the upper bound current is inversely proportional to the charging time and SEI layer resistance. To get the same charging time for both methodologies, the maximum current limit of SEI optimal strategy should be higher than optimal CCCV. Two cases are recorded in this analysis, where charging time is the same in both strategies.

It is evident from Table 1 that in order to get the same charging time, current upper bounds in both strategies are not the Table 1: Same Charging Time Cases

\begin{tabular}{|c|c|c|c|}
\hline $\begin{array}{c}\text { Case } \\
\text { No. }\end{array}$ & $\begin{array}{c}\text { Charging } \\
\text { time (s) }\end{array}$ & $\begin{array}{c}\text { Current upper bound in } \\
\text { SEI optimal charging, } I_{\max }(\mathrm{A})\end{array}$ & $\begin{array}{c}\text { Current upper bound in } \\
\text { optimal CCCV charging, } I_{\max }(\mathrm{A})\end{array}$ \\
\hline 1 & 1125 & 5 & 4.55 \\
\hline 2 & 902 & 7 & 5.7 \\
\hline
\end{tabular}

same. Consider case 2 of Table 1, the charging time is set to 902 seconds which is charging time for SEI optimal strategy at $I_{\max }=7 \mathrm{~A}$ (Figure 1(d), (e) and (f)). To get same charging time for optimal CCCV, current upper bound needs to decrease because at $I_{\max }=7 \mathrm{~A}$, charging time is 740 seconds. Figures 3 and 4 show the relationship between SOC, current and SEI layer resistance versus time.

Figure 3(a) shows the SOC of the negative electrode. Reference SOC is attained in both methods at the same time. The optimal SOC profile initially has a slightly low slope as compared to optimal CCCV. This is because of the optimal charge current profile (Figure 3(b)), which starts at a reasonably low value. The primary reason is to control side reaction overpotential at a low state of charge, ultimately avoiding the effects of lithium plating. 


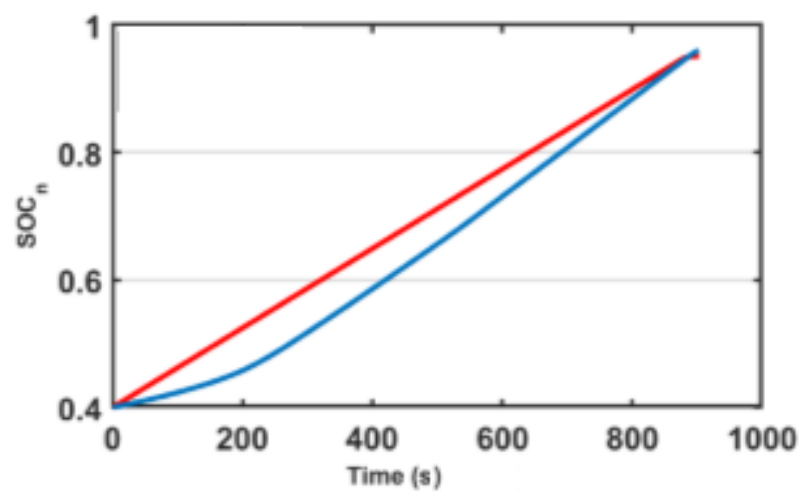

(a)

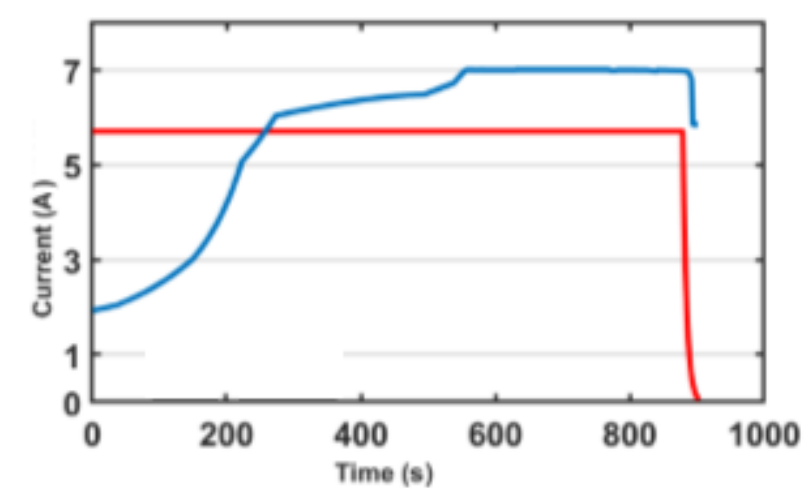

(b)

Figure 3: State of charge (a) and current profile (b) for same charging time; Case 2 of Table 1 Optimal CCCV Charging $\left(I_{\max }=5.7 A\right)$, Proposed SEI optimal charging $\left(I_{\max }=7 A\right)$

At $I_{\max }=5.7 \mathrm{~A}$, SEI layer resistance is recorded as $0.01155 \Omega \mathrm{m}^{2}(15.5 \%$ increase $)$ compared to the SEI layer growth in the proposed SEI optimal charging method, as shown in Figure 4. The percentage increase in the SEI layer at $I_{\max }=5.7 \mathrm{~A}$, goes up to approximately $2.5 \%$ compared to charging at $I_{\max }=7 \mathrm{~A}$. Hence, the percentage difference of SEI layer resistance between proposed SEI optimal and optimal CCCV strategies climbs up to $8.6 \%$ keeping similar charging time.

Similar results are found for case 1 of Table 1, which shows that to get the similar charging time of 1125 seconds, the current upper bounds must be $5 A$ and $4.55 A$ in proposed SEI optimal and optimal CCCV strategies, respectively. The SEI layer resistance in optimal CCCV is increased to $0.01211 \Omega m^{2}(21.1 \%$ increase $)$ which is $0.0118 \Omega m^{2}$ at $I_{\max }=5 \mathrm{~A}$. An overall percentage increase of $3.1 \%$ is recorded as at $I_{\max }=4.55 \mathrm{~A}$ compared to optimal CCCV at $I_{\max }=5 \mathrm{~A}$. Thus, the percentage difference of SEI layer resistance between proposed SEI optimal and optimal CCCV strategies climbs up to $7.8 \%$ keeping similar charging time.

Scenario I analysis is summarised in Figure 5. It shows a relationship between charging time versus maximum current upper bound. Maximum current is taken from $2.3 A(1 C)$ to $9.2 A(4 C)$. Understandably, optimal CCCV charging is fast at a specific current upper bound value. For example, at $I_{\max }=7 \mathrm{~A}$, proposed SEI optimal charging takes almost three extra minutes. In order to find the same charging time for both methods, one can get values of current upper bounds by drawing vertical and horizontal lines from data labels. For charging time of 1100 seconds, maximum current values of optimal CCCV 


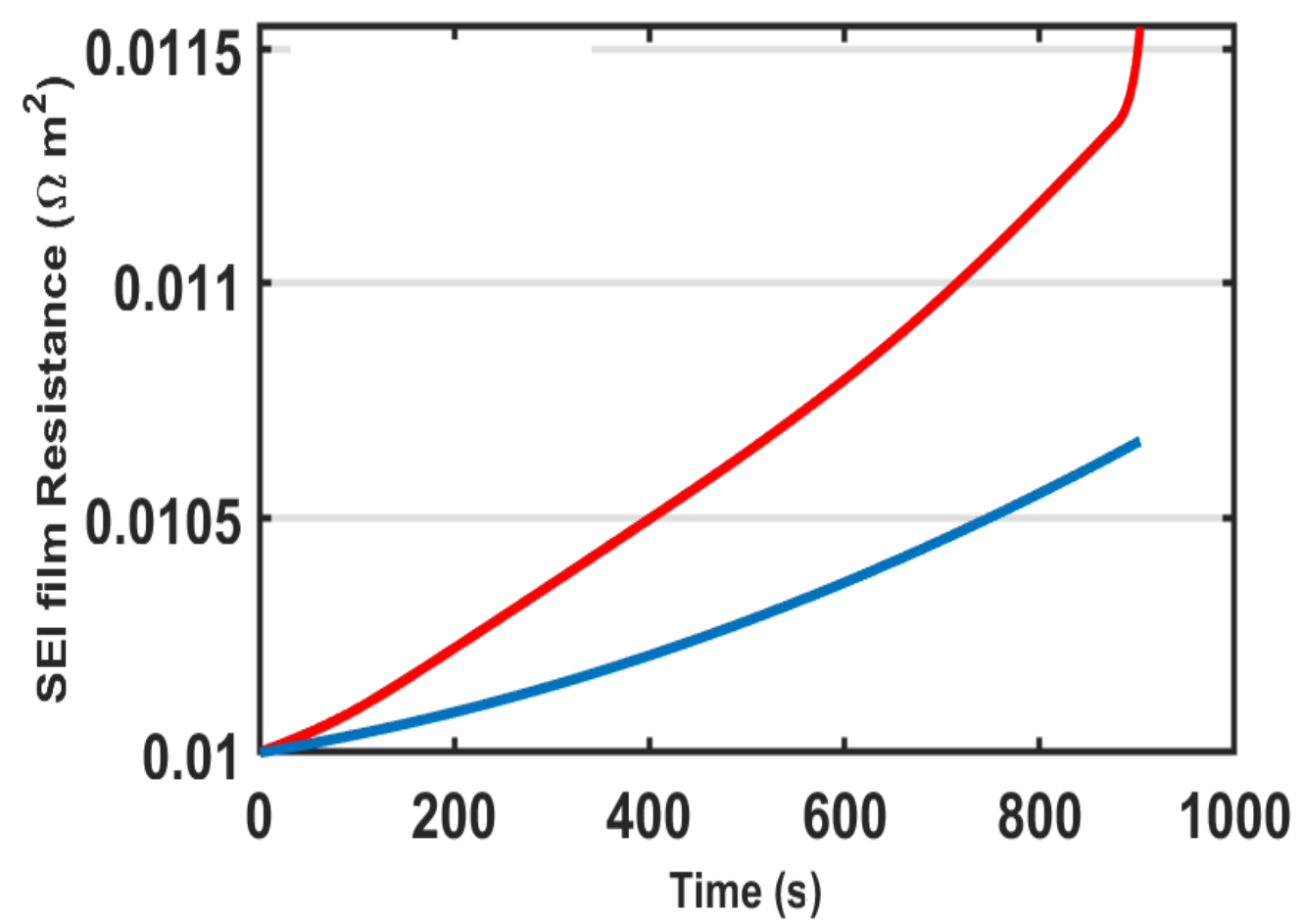

Figure 4: Same Charging time: Comparison of SEI film resistance in optimal CCCV charging $\left(I_{\max }=5.7 A\right)$ and proposed SEI optimal charging $\left(I_{\max }=5.7 \mathrm{~A}\right)$

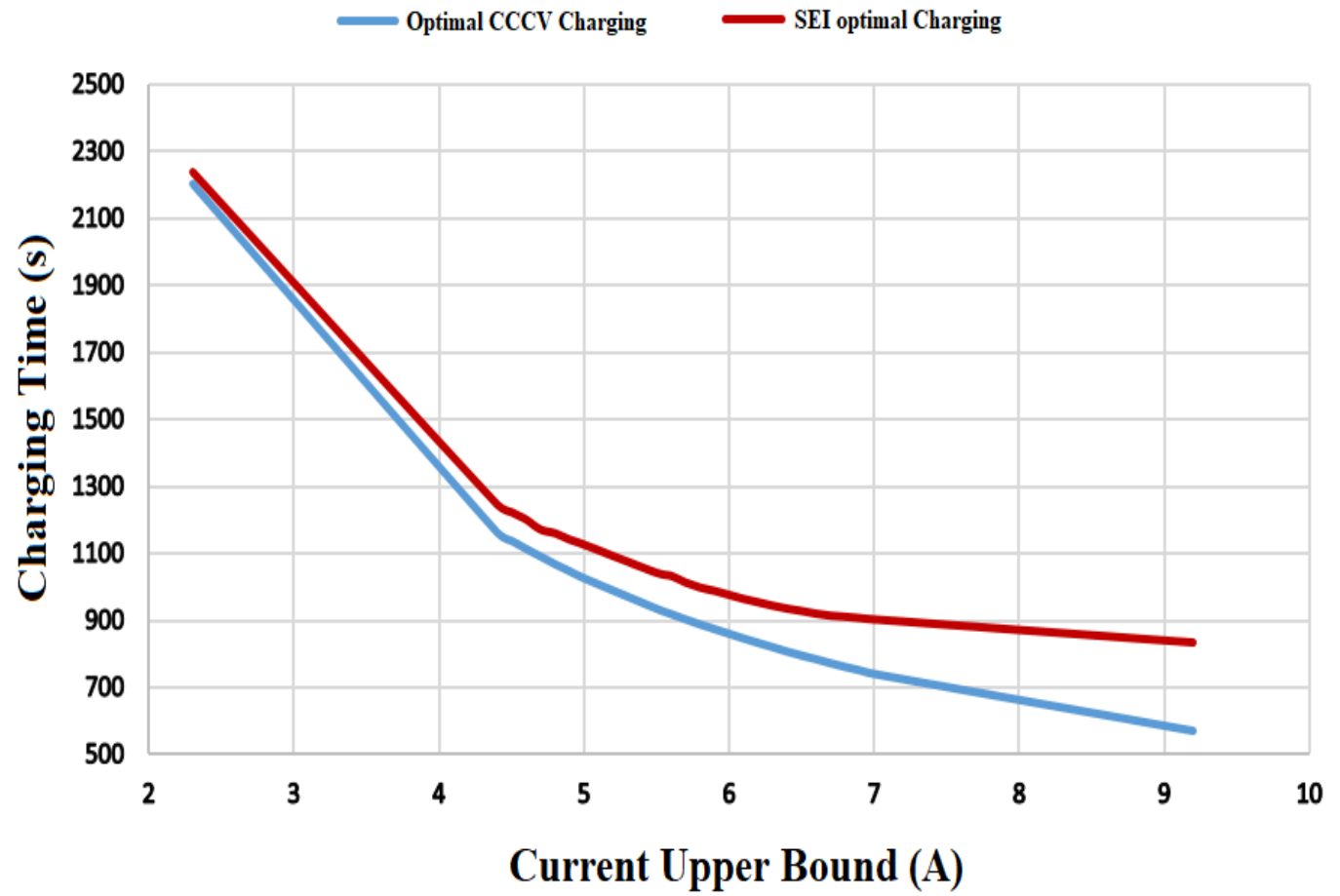

Figure 5: Relationship between charging time and current upper bound $\left(I_{\max }\right)$ in optimal CCCV and proposed SEI optimal charging methodologies

and proposed SEI optimal methodologies should be $4.7 \mathrm{~A}$ and $5.1 \mathrm{~A}$, respectively. As the maximum value of current increases, charging time difference between optimal CCCV and the proposed SEI optimal charging strategies also increases.

The conclusion from the scenario I is that charging time for proposed SEI optimal and optimal CCCV strategies can be the same, but on the cost of higher SEI layer growth. The proposed method outperforms the optimal CCCV because of two 
reasons; $(i)$ SEI layer growth is low and $(i i)$ it successfully avoids side reaction overpotential to attain value less than zero. Thus by keeping current upper bound or charging time same, the proposed SEI optimal framework is far better than optimal CCCV as it minimises SEI layer growth and tackles lithium plating too.

7.2. Scenario II: Same SEI layer Resistance

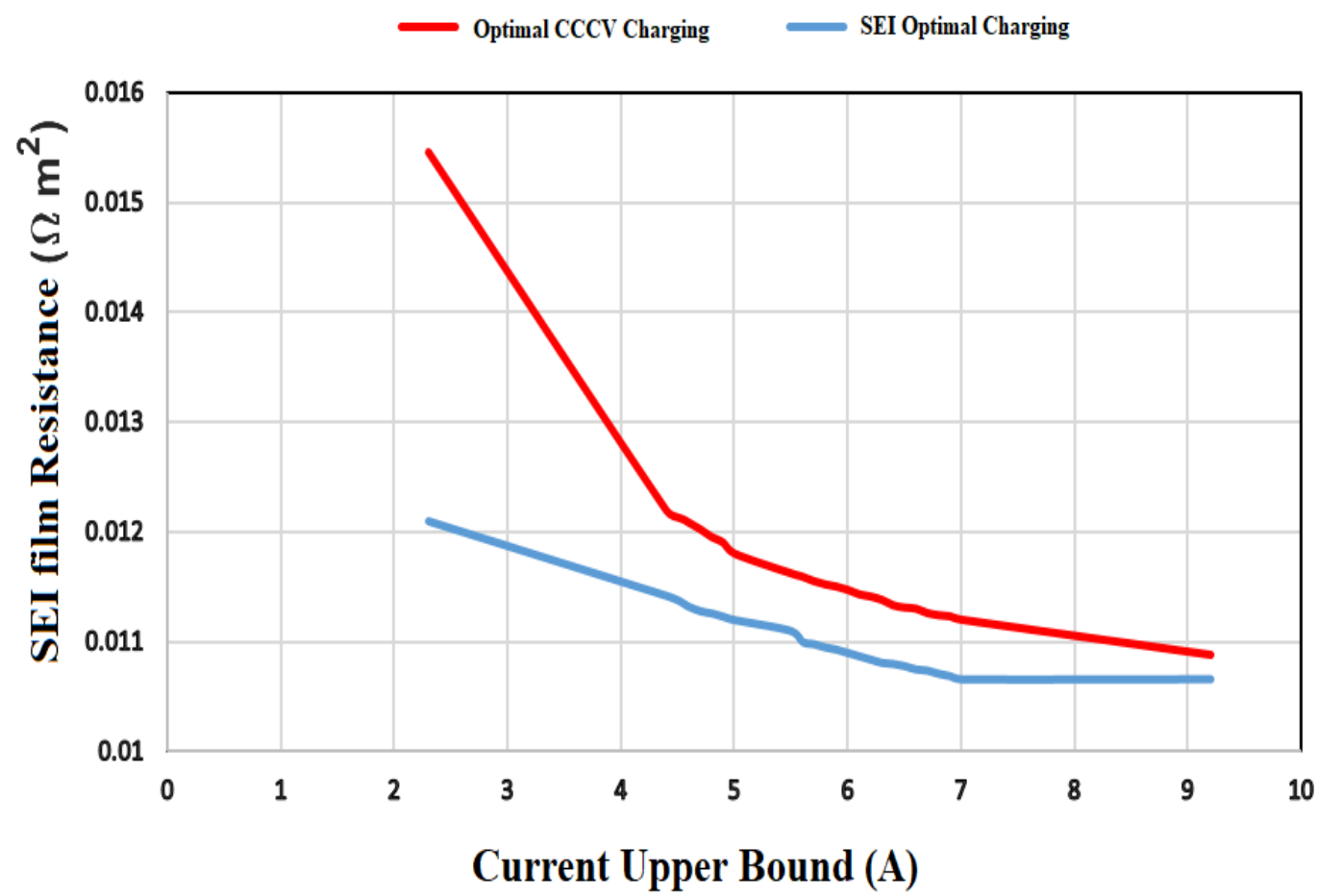

Figure 6: Relationship between SEI film resistance and current upper bound $\left(I_{\max }\right)$ in optimal CCCV and proposed SEI optimal charging methodologies

Figure 6 shows the relationship between SEI layer growth and maximum current in both methodologies. It can be seen that at low current rating, the difference in the SEI layer resistances corresponding to optimal CCCV and proposed SEI optimal strategies is high. As the value of the maximum current increases, the difference in the SEI layer resistances decreases. The primary reason for a more significant difference in the SEI layer at low current, is the high charging time. To get SEI layer resistance of $0.011 \Omega \mathrm{m}^{2}$, the maximum current in optimal CCCV and proposed SEI optimal methodologies should be $8.1 \mathrm{~A}$ and 5.6A, respectively. Thus, a proposed optimal framework uses a low current upper bound along with generating small value of SEI layer resistance. It has been noted that there is no significant change in SEI layer resistance from $I_{\max }=7 \mathrm{~A}$ to $9.2 \mathrm{~A}$ in proposed optimal charging framework.

The percentage difference in SEI layer resistance between optimal CCCV and proposed SEI Optimal methodologies is shown in Figure 77. The highest percentage difference is recorded as $24 \%$ at the current rating of $1 C$. This difference kept on decreasing from $1 C$ to $2 C$ and $3 C$ to $4 C$. The percentage difference fluctuates around $5 \%$ from $2 C$ to $3 C$. Because of the minimum range of work from $3 C$ to $4 C$, \% difference in SEI layer growth is almost constant.

Charging time is not the only factor that influences SEI layer growth, but higher current can contribute to exfoliation of graphite. It leads to a loss of active anode material which can be a source of capacity and power fade. Hence, it can be concluded from the above analysis that battery charging is a trade-off between optimal charging time and the current upper 


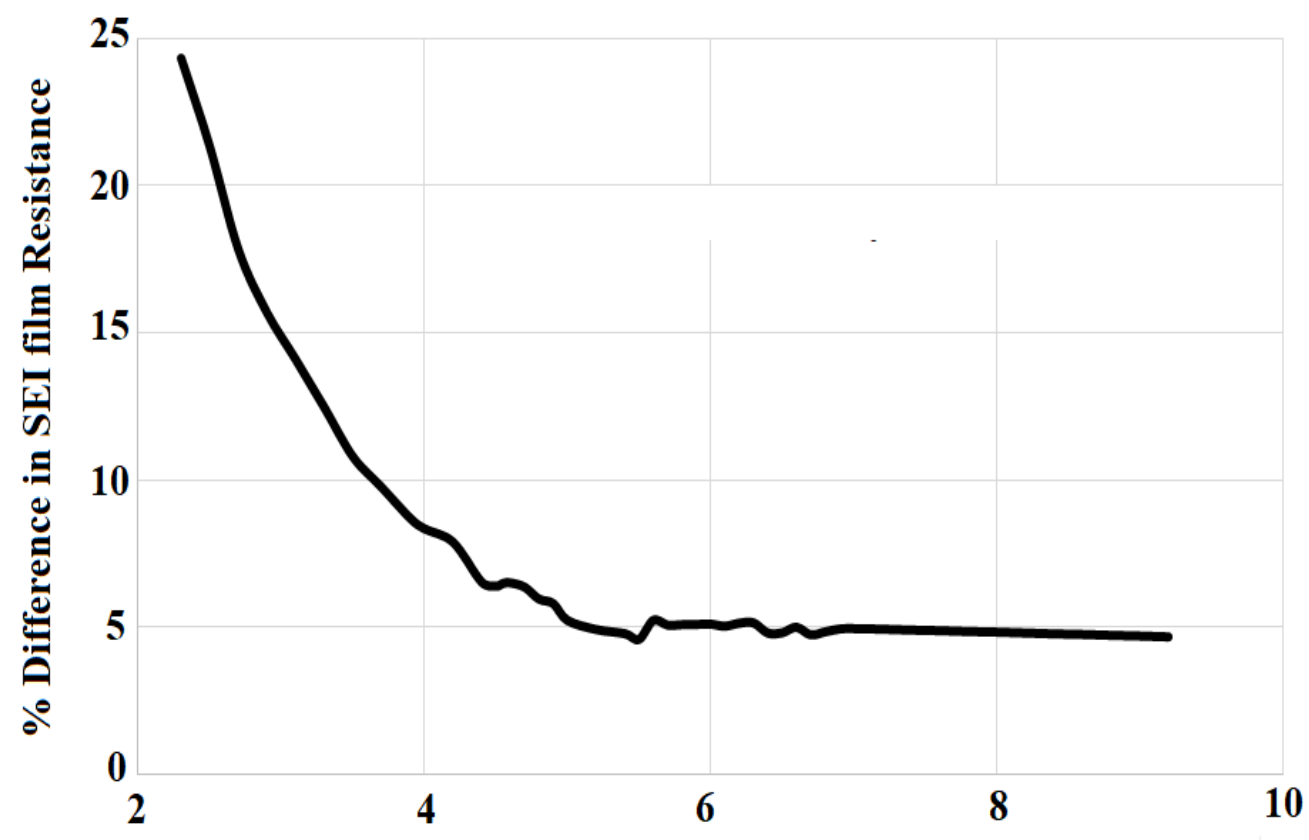

Current Upper Bound (A)

Figure 7: Percentage difference in SEI layer resistances between optimal CCCV and proposed SEI optimal charging methodologies from $I_{m a x}=1 \mathrm{C}$ $(2.3 \mathrm{~A})$ to $I_{\max }=4 \mathrm{C}(9.2 \mathrm{~A})$

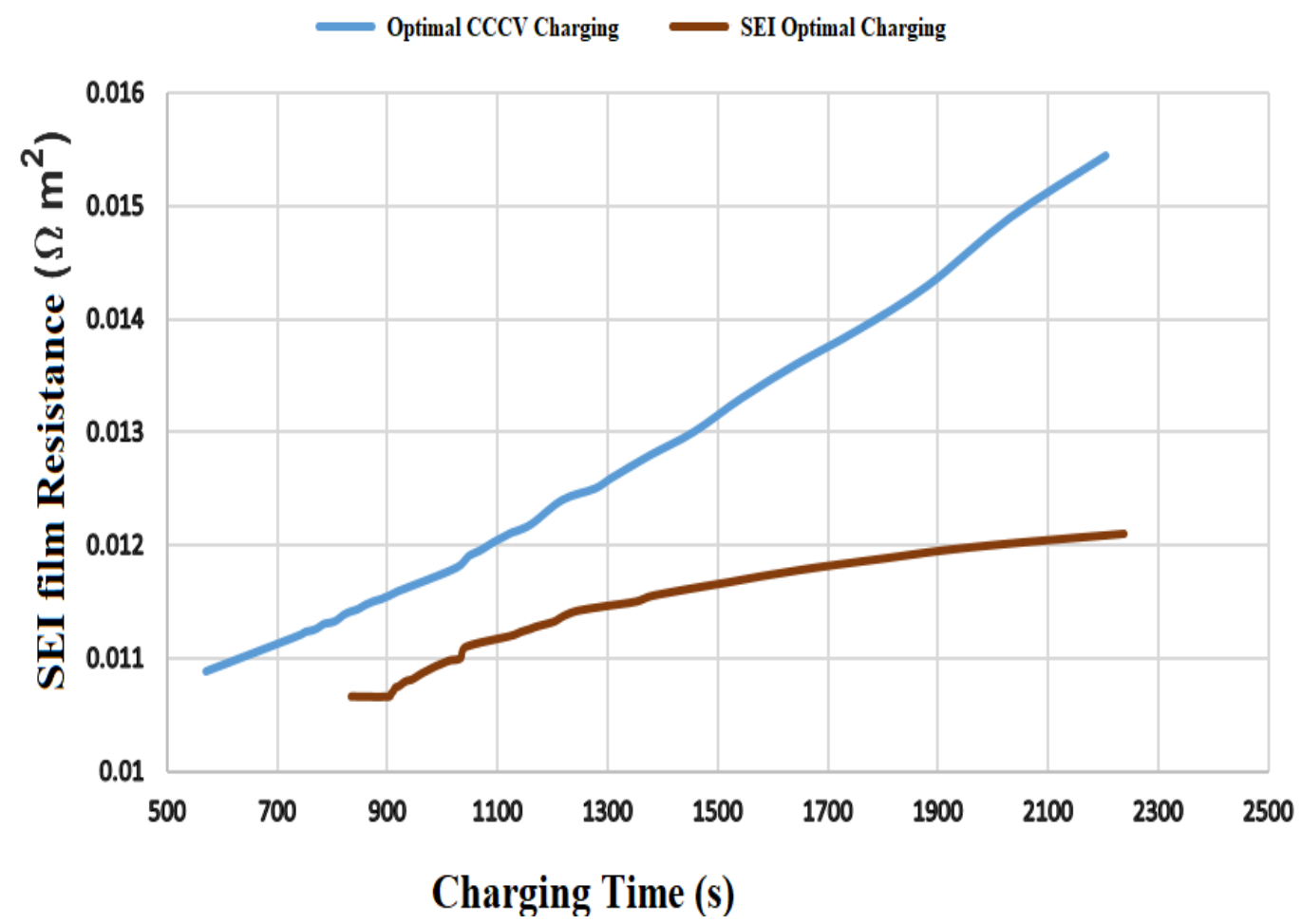

Figure 8: SEI film resistance versus charging time in optimal CCCV and proposed SEI optimal charging methodologies

bound.

Scenario II analysis is summarised in Figure 8. It shows the relationship between SEI layer resistance and charging time at the current upper bound range of $1 C$ to $4 C$. It is evident from Figure 8 that at any charging time, SEI layer resistance is higher in optimal CCCV than the proposed SEI optimal charging. SEI layer resistance is recorded as $0.0117 \Omega m^{2}$ (proposed 
Table 2: Overall computational times of optimal CCCV and proposed SEI optimal charging strategies at different current rates

\begin{tabular}{|c|c|c|}
\hline Current Upper Bound $\left(I_{\max }\right)$ & CCCV $(\mathrm{s})$ & NMPC $(\mathrm{s})$ \\
\hline $1 \mathrm{C}(2.3 \mathrm{~A})$ & 242 & 285 \\
\hline $2 \mathrm{C}(4.6 \mathrm{~A})$ & 132 & 164 \\
\hline $3 \mathrm{C}(6.9 \mathrm{~A})$ & 54 & 78 \\
\hline $4 \mathrm{C}(9.2 \mathrm{~A})$ & 39 & 58 \\
\hline
\end{tabular}

SEI optimal) and $0.0132 \Omega \mathrm{m}^{2}$ (optimal CCCV), at the charging time of 1500 seconds. The percentage difference in SEI layer resistance is $12 \%$ whereas the corresponding maximum current $I_{\max }$ is $3.2 A$ and $3.6 A$ in the proposed SEI optimal and optimal CCCV strategies, respectively.

Another critical aspect of online control strategies is computational time. Although the proposed NMPC successfully minimises the SEI layer resistance, it must be practically implementable. Computational times of both optimal and CCCV methodologies are presented in Table 2 at different maximum current upper bounds using MATLAB R2016b on DELL laptop with intel (R) Core (TM) i-7-8650U CPU @ 1.90GHz 2.11 GHz processor. The simulations of optimisation problems (39) and (46) are conducted using 4 collocation points, same SPM model and a prediction horizon of 200 seconds. Moreover, a more significant range of SOC (10\% to $96 \%)$ is considered to evaluate the full charging process. Overall computational time is defined as the time taken by an algorithm to charge the battery from initial to final SOC. The overall computational times of optimal CCCV and the proposed SEI optimal strategies at 1C rating are 242 and 285 seconds, respectively. The percentage difference in computational times between both strategies tends to increase as the current rate increases. The higher current decreases the simulation time, keeping all other parameters constant. At $1 \mathrm{C}$, the difference in computational time between both strategies is 43 seconds. The difference keeps on decreasing as current upper bound increases, 19 seconds at 4C. As computational time difference between two strategies is not significant; it can be concluded that the proposed SEI optimal charging strategy is suitable for real-time implementation in battery management systems.

\section{Conclusion}

This article proposes an online non-linear model predictive control framework which minimises SEI layer film resistance during charging. It uses the integral Gauss pseudo-spectral approach to optimise battery charging trajectory. Apart from the SEI layer minimisation, it deals with another side reaction, i.e. lithium plating, which is the main factor causing capacity fade. The proposed algorithm guarantees that the battery works in a healthy regime during charging. It is evident from the results that SEI film resistance decreases significantly in proposed SEI optimal charging as compared to optimal CCCV charging. There is up to $24 \%$ difference in SEI layer growth, recorded in case of proposed SEI optimal methodology. In case of same charging time for both charging methodologies, SEI layer resistance is higher in optimal CCCV charging. The proposed SEI optimal methodology is the best candidate to charge the battery. This work can be further used to compute cyclic capacity fade, thus estimating the life of batteries. 


\section{Nomenclature}

\section{List of Symbols}

c concentration $\left(\mathrm{mol} \mathrm{m} \mathrm{m}^{-3}\right.$ )

$r$ radius of electrode $(m)$

$t \quad$ time $(s)$

$J \quad$ molar flux $\left(A m^{-2}\right)$

$F \quad$ Faraday's constant $\left(C \mathrm{~mol}^{-1}\right)$

$D$ Diffusion constant $\left(m^{2} s^{-1}\right)$

$a \quad$ interfacial surface area $\left(m^{-1}\right)$

$I \quad$ current $(A)$

$S$ area of electrode $\left(m^{2}\right)$

$L \quad$ length of electrode $(m)$

$T$ temperature $\left({ }^{\circ} \mathrm{C}\right)$

$i \quad$ current density $\left(A m^{-2}\right)$

$U$ equilibrium potential $(V)$

$V \quad$ voltage of cell $(V)$

$M$ molecular weight $\left(\mathrm{kg} \mathrm{mol}^{-1}\right)$

Greek

$\epsilon$ porosity of electrode

$\alpha$ transfer coefficient

$\eta \quad$ overpotential

$\phi \quad$ potential, Legendre polynomial

$\beta \quad$ time coefficient

$\delta \quad$ thickness

$\rho \quad$ density of products

$\kappa \quad$ conductivity of electrolyte 


$$
\begin{array}{cl}
s & \text { solid state } \\
p & \text { positive electrode } \\
n & \text { negative electrode } \\
\text { avg } & \text { average } \\
\text { max } & \text { maximum } \\
\text { surf } & \text { surface } \\
a & \text { anode } \\
c & \text { cathode } \\
g & \text { gas } \\
1 & \text { solid } \\
2 & \text { solution } \\
f i l m & \text { SEI film } \\
\text { sr } & \text { side reaction } \\
\text { SEI } & \text { solid electrolyte interface } \\
e q & \text { equivalent } \\
r e f & \text { reference }
\end{array}
$$

\section{Acronyms}

$\begin{array}{cl}\text { NMPC } & \text { non-linear model predictive control } \\ \text { EV } & \text { electric vehicle } \\ \text { CCCV } & \text { constant current constant voltage } \\ \text { SOH } & \text { state of health } \\ \text { BMS } & \text { battery management system } \\ \text { NLP } & \text { non-linear programming } \\ \text { SPM } & \text { single particle model } \\ \text { SOC } & \text { state of charge } \\ \text { MPC } & \text { model predictive control } \\ \text { IAM } & \text { integration approximation matrix }\end{array}$

\section{References}

[1] Christopher D Rahn and Chao-Yang Wang. Battery systems engineering. John Wiley \& Sons, 2013.

[2] Nalin A Chaturvedi, Reinhardt Klein, Jake Christensen, Jasim Ahmed, and Aleksandar Kojic. Modeling, estimation, and control challenges for lithium-ion batteries. In Proceedings of the 2010 American Control Conference, pages $1997-2002$. IEEE, 2010.

[3] Saeed Khaleghi Rahimian, Sean Rayman, and Ralph E White. Optimal charge rates for a lithium ion cell. Journal of Power Sources, 196(23):10297-10304, 2011. 
[4] Huazhen Fang and Yebin Wang. Health-aware and user-involved battery charging management for electric vehicles using linear quadratic control. In ASME 2015 Dynamic Systems and Control Conference, pages V001T13A009-V001T13A009. American Society of Mechanical Engineers, 2015.

[5] Scott J Moura, Joel C Forman, Saeid Bashash, Jeffrey L Stein, and Hosam K Fathy. Optimal control of film growth in lithium-ion battery packs via relay switches. IEEE Transactions on Industrial Electronics, 58(8):3555-3566, 2010.

[6] Saeid Bashash, Scott J Moura, Joel C Forman, and Hosam K Fathy. Plug-in hybrid electric vehicle charge pattern optimization for energy cost and battery longevity. Journal of power sources, 196(1):541-549, 2011.

[7] Abdullah-Al Mamun, Iyswarya Narayanan, Di Wang, Anand Sivasubramaniam, and Hosam K Fathy. Multi-objective optimization to minimize battery degradation and electricity cost for demand response in datacenters. In ASME 2015 Dynamic Systems and Control Conference, pages V002T26A004-V002T26A004. American Society of Mechanical Engineers, 2015.

[8] Xiaosong Hu, Shengbo Li, Huei Peng, and Fengchun Sun. Charging time and loss optimization for linmc and lifepo4 batteries based on equivalent circuit models. Journal of Power Sources, 239:449-457, 2013.

[9] Ji Liu, Guang Li, and Hosam K Fathy. An extended differential flatness approach for the health-conscious nonlinear model predictive control of lithium-ion batteries. IEEE Transactions on Control Systems Technology, 25(5):1882-1889, 2017.

[10] Ji Liu, Guang Li, and Hosam K Fathy. A computationally efficient approach for optimizing lithium-ion battery charging. Journal of Dynamic Systems, Measurement, and Control, 138(2):021009, 2016.

[11] JC Burns, DA Stevens, and JR Dahn. In-situ detection of lithium plating using high precision coulometry. Journal of The Electrochemical Society, 162(6):A959-A964, 2015.

[12] Changfu Zou, Xiaosong Hu, Zhongbao Wei, Torsten Wik, and Bo Egardt. Electrochemical estimation and control for lithium-ion battery health-aware fast charging. IEEE Transactions on Industrial Electronics, 65(8):6635-6645, 2017.

[13] Nan Li, Feng Gao, Tianqu Hao, Zhan Ma, and Chenghui Zhang. SOH balancing control method for the MMC battery energy storage system. IEEE Transactions on Industrial Electronics, 65(8):6581-6591, 2017.

[14] Kaiyuan Li, Feng Wei, King Jet Tseng, and Boon-Hee Soong. A practical lithium-ion battery model for state of energy and voltage response prediction incorporating temperature and ageing effects. IEEE Transactions on Industrial Electronics, 65(8):6696-6708, 2017.

[15] P Ramadass, Bala Haran, Parthasarathy M Gomadam, Ralph White, and Branko N Popov. Development of first principles capacity fade model for li-ion cells. Journal of the Electrochemical Society, 151(2):A196-A203, 2004.

[16] Michelle A Kehs, Michael D Beeney, and Hosam K Fathy. Computational efficiency of solving the dfn battery model using descriptor form with legendre polynomials and galerkin projections. In 2014 American Control Conference, pages 260-267. IEEE, 2014. 
[17] Venkatasailanathan Ramadesigan, Paul WC Northrop, Sumitava De, Shriram Santhanagopalan, Richard D Braatz, and Venkat R Subramanian. Modeling and simulation of lithium-ion batteries from a systems engineering perspective. Journal of the electrochemical society, 159(3):R31-R45, 2012.

[18] Chris Manzie, Changfu Zou, and Dragan Nesic. Simplification techniques for pde-based li-ion battery models. In 2015 54th IEEE Conference on Decision and Control (CDC), pages 3913-3921. IEEE, 2015.

[19] M. Safari and C. Delacourt. Modeling of a commercial graphite/LiFePO4 cell. Journal of The Electrochemical Society, 158(5):A562, 2011.

[20] Guodong Fan and Marcello Canova. Model order reduction of electrochemical batteries using galerkin method. In ASME 2015 Dynamic Systems and Control Conference, pages V001T13A006-V001T13A006. American Society of Mechanical Engineers, 2015.

[21] Doron Aurbach. Review of selected electrode-solution interactions which determine the performance of li and li ion batteries. Journal of Power Sources, 89(2):206-218, 2000.

[22] I Nainville, A Lemarchand, and J-P Badiali. Passivation of a lithium anode: A simulation model. Electrochimica acta, 41(18):2855-2863, 1996.

[23] D Aurbach, B Markovsky, I Weissman, E Levi, and Y Ein-Eli. On the correlation between surface chemistry and performance of graphite negative electrodes for li ion batteries. Electrochimica acta, 45(1-2):67-86, 1999.

[24] M Safari, M Morcrette, A Teyssot, and C Delacourt. Multimodal physics-based aging model for life prediction of li-ion batteries. Journal of The Electrochemical Society, 156(3):A145-A153, 2009.

[25] Geoffrey Todd Huntington. Advancement and analysis of a Gauss pseudospectral transcription for optimal control problems, volume 69. Citeseer, 2007.

[26] Josef Stoer and Roland Bulirsch. Introduction to numerical analysis, volume 12. Springer Science \& Business Media, 2013.

[27] Owe Axelsson. Global integration of differential equations through lobatto quadrature. BIT Numerical Mathematics, $4(2): 69-86,1964$.

[28] David Benson. A Gauss pseudospectral transcription for optimal control. PhD thesis, Massachusetts Institute of Technology, 2005. 